\title{
Autophagy in Tumorigenesis and Cancer Treatment
}

\author{
Dong-Wei $\mathrm{Xu}^{1}$, Guan-Qing Zhang1, Zong-Wei Wang ${ }^{2}$, Xiao-Yin $\mathrm{Xu}^{3 *}$, Tong- \\ Xiang Liu ${ }^{1,3 *}$
}

\begin{abstract}
Autophagy is a self-digestion process, wrapping cytoplasmic proteins or organelles to form vesicles for degradation in lysosomes. The process plays an important role in the maintenance of intracellular homostasis. Here we overview articles on autophagy and cancer/tumors in Pubmed and found 327 articles. Autophagy exists in many tumors and is involved in cell malignant transformation and tumor cell growth. In early phases of tumorigenesis, autophagy clears the abnormally folded proteins and dysfunctional organelles such as mitochondria. Autophagy can also inhibit cell stress responses and prevent genetic damage. When a tumor develops, autophagy helps tumor cells survive nutritional deficiencies and hypoxic conditions. Studies of autophagy in the occurrence and progression of tumors should provide new therapeutic strategies for tumors.
\end{abstract}

Keywords: Autophagy - regulatory mechanism - signaling pathway - tumor - cancer treatment

Asian Pac J Cancer Prev, 16 (6), 2167-2175

\section{Introduction}

In 1955, de Duve et al. (1955) first described the term "autophagy" to distinguish lysosomal degradation, or cellular "eating" (phagy) of self (auto), from the breakdown of extracellular material (heterophagy). Autophagy is a cytoplasmic, homeostatic process by which cells degrade their interior components, including targets that are too large for other degradative systems, in response to external and internal triggers(Mizushima et al., 2008). With the establishment of the model of autophagy in yeast and the development of gene technology, research on autophagy has been progressing rapidly over the last several decades. Autophagy is not only important to cell growth and differentiation and stress response to the environment but also plays a critical role in preventing some diseases such as cancer and neurodegenerative conditions as well as provides protection against infection of pathogenic microorganisms (White et al., 2011).

Type I programmed cell death (apoptosis) and necrosis are known and widely accepted as two types of cell death in mammals (Galluzzi et al., 2012). Type II programmed cell death (autophagy) is a cellular reuse of their own unnecessary or damaged organelles and macromolecular components, and generates energy and metabolites. Autophagy is a kind of adaptive response to sublethal stress (Hotchkiss et al., 2009) and it allows cells that are in a hungry status or lack of growth factor to survive temporarily. However the cells that cannot obtain nutrition over the long term will die eventually (autophagy correlation cell death). Therefore autophagy has been identified as the third kind of mode of cell death (Levine et al., 2008). In recent years, the role of autophagy in cancer attracts more attention and likely to offer a new venue of developing novel treatment of cancer.

\section{Definition and Three Kinds of Cell Autophagy}

Autophagy was originally used to describe the adaptive response under the electron microscope, which exist in multiple stages of cell disruption. Single or double membrane structure of vacuoles fuse with lysosomes, then encircle and digest cytoplasmic components (including organelles) (Gozuacik et al., 2004; Klionsky, 2007). There are three major types of autophagy in eukaryotic cells-macroautophagy, microautophagy, and chaperonemediated autophagy (CMA)-and they are mechanistically different from each other (Massey et al., 2004; Klionsky, 2005) (Figure 1). Both macro- and microautophagy involve dynamic membrane rearrangement to engulf portions of the cytoplasm, and they have the capacity for the sequestration of large structures, such as entire organelles. Microautophagy involves the direct engulfment of cytoplasm at the lysosome surface by invagination, protrusion, and septation of the lysosome membrane. In contrast, during macroautophagy, portions of cytoplasm are sequestered into a de novo-formed double-membrane vesicle termed an autophagosome. Subsequently, the completed autophagosome fuses with the lysosome/ vacuole and the inner single-membrane vesicle is released 
Dong-Wei Xu et al

into the lumen. In either case, the membrane of the resulting autophagic body is lysed to allow the contents to be broken down, and the resulting macromolecules are transported back into the cytosol through membrane permeases for reuse. In contrast, CMA does not involve a similar type of membrane rearrangement; instead, it translocates unfolded, soluble proteins directly across the limiting membrane of the lysosome (Yang et al., 2009).

\section{Autophagy-related Genes (ATG)}

Due to the advantage of yeast as a powerful genetic system, researchers have identified 38 autophagy-related genes(ATG) that are specifically involved in autophagy (Huang et al., 2007; Geng et al., 2008; Klionsky, 2014). The autophagy process can be dissected into a series of steps: induction, vesicle nucleation, cargo recognition (for specific types of autophagy) and packaging, vesicle expansion and completion, ATG protein cycling, vesicle fusion with the lysosome/vacuole, vesicle breakdown and nutrient recycling. The ATG proteins can be categorized into different groups according to their functions at the various steps of the pathway. For example, the ATG1 kinase complex is involved in induction (although it also acts at later stages), ATG6 and ATG14 participate in vesicle nucleation, ATG11 and ATG19 are required for cargo recognition and packaging, the ATG8 and ATG12 ubiquitin-like conjugation systems are involved in vesicle formation, and ATG9, ATG23 and ATG27 participate in the $15-17$ protein retrieval step(Ohsumi, 2006) . Although the majority of the ATG proteins have probably been identified, their exact functions are largely unknown. Continued investigation of the molecular machinery in yeast will provide useful information that will be applicable for studying this process in other organisms. The process of autophagy in higher eukaryotes is essentially the same as that in yeast and mammalian orthologs of many of the yeast ATG genes have been identified. Many homologous ATG genes have been found in mammals. The naming of mammalian autophagy genes and yeast are similar, but there are individual differences, such as yeast ATG8 corresponding mammals LC3, yeast ATG6 corresponding Beclin 1 of mammals. Mammalian autophagy related genes are shown in Table 1 (Klionsky et al., 2003; Tanida et al., 2004).

\section{The Involvement of ATGs and their Signaling Pathway in Tumorigenesis}

Autophagy is highly regulated and under the control of a number of signaling pathways. The following is a summary of recent research on the regulation of autophagy.

\section{PI3K-AKT-mTOR pathway}

The PI3K-AKT-mTOR pathway (Figure 2) plays an important role in autophagy induction. Activated PI3K generates phosphatidylinositol-3,4,5-triphosphate, which recruits PDK1 and AKT serine/threonine kinase at the plasma membrane. This results in activation of AKT. AKT activates multiple downstream target, including the mTOR pathway. AKT in this patyway is flanked by two

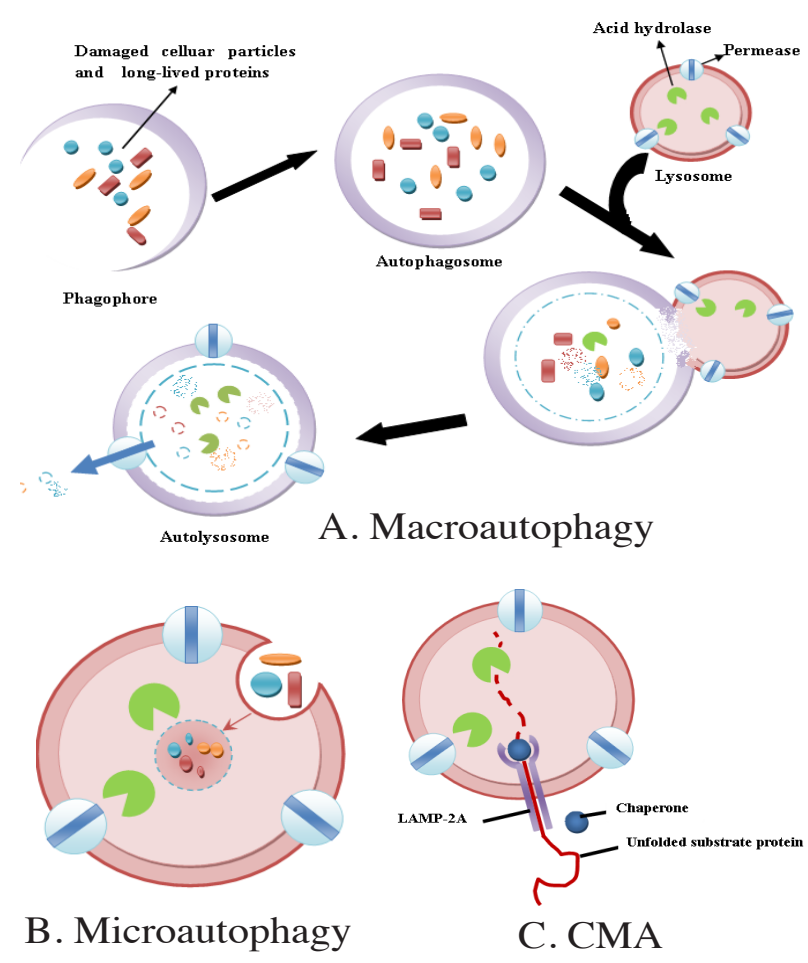

Figure 1. Three Main Types of Autophagy. The schematic depicts these processes in lower and higher eukaryotes. For example, the lysosome is much smaller than the fungal vacuole. Also, chaperone-mediated autophagy has only been characterized in higher eukaryotes, whereas microautophagy and macroautophagy are evolutionarily conserved. Macroautophagy is the best-characterized pathway out of the three and the hallmark of this process is the formation of a double-membrane vesicle that non-selectively sequesters cytoplasmic components and delivers them to the lysosome or vacuole for degradation and recycling of the cargo

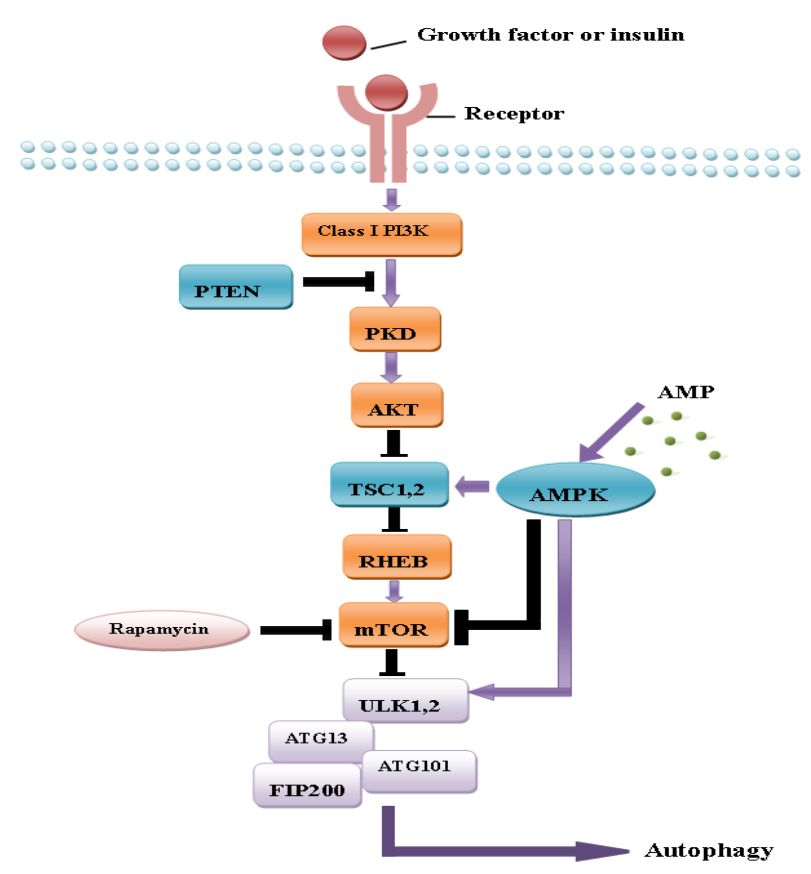

Figure 2. PI3K-AKT-mTOR Pathway: Proteins depicted in orange and blue ultimately inhibit and stimulate, respectively, the activity of the ATG1 kinase complex (the ULK1 and ULK2 complex in mammals) and subsequently, autophagy 
Table 1. Mammalian Autophagy Related Genes

\begin{tabular}{lll}
\hline Yeast & \multicolumn{1}{c}{ Human } & \multicolumn{1}{c}{ Mouse } \\
\hline ATG1 & ULK1 & mATG3/mAPG3 \\
ATG3 & hATG3/hAPG3 & \\
ATG4 & hATG4A/HsATG4A/ & \\
& HsAPG4A/autophagin-2 & \\
& hATG4B/HsATG4B/ & \\
& hAPG4B/autophagin-1 & \\
& hATG4C/HsAUTL1/ & \\
& autophagin-3 & \\
ATG5 & hAPG4D/autophagin-4 & \\
ATG6 & hATG5/hAPG5 & \\
ATG7 & hATG7/HsGSA7/hAPG7 & mATG7/mAPG7 \\
ATG8 & LC3 & \\
ATG10 & & mATG10/mAPG10 \\
ATG12 & hATG12/hAPG12 & mATG12/mAPG12 \\
ATG14 & ATG14L & \\
ATG16 & & ATG16L/APG16L \\
\hline
\end{tabular}

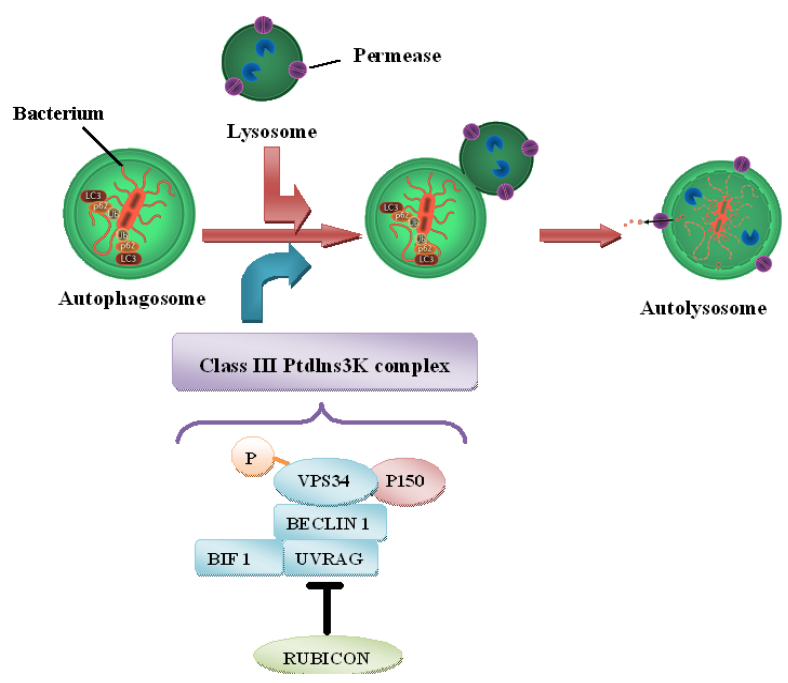

Figure 3. The Vps34 Complex in Autophagosome Formation and Maturation

tumor suppressors, PTEN and TSC1/TSC2 heterodimer. The PI3K-AKT-mTOR axis, a vital process for initiating theautophagy pathway, regulates several biological events including cell cycle and proliferation and is mutated in many human malignancies (Maiuri et al., 2009; Morselli et al., 2009). In tumorigenesis, growth signaling constitutively activates RTKs, which then activate Rheb and PI3K. Rheb is a small GTP-binding protein that activates mTOR in its GTP-bound form, whereas Rheb and PI3K converge to activate mTOR to stimulate cell growth and inhibit autophagy (Ghayad et al., 2010). Because most cancers can harbor activating mutations of master regulators, such as TSC1, TSC2, AKT, and ribosomal S6 kinase (RSK) (Inoki et al., 2009), many cancers exhibit enhanced activation of mTOR and inhibit autophagy.The PI3K-AKT-mTOR pathway is a frequently hyperactivated pathway in cancer and is important for tumor cell growth and survival. The development of targeted therapies against mTOR, a vital substrate along this pathway, led to the approval of allosteric inhibitors, including everolimus and temsirolimus, for the treatment of breast, renal, and
Importance of Autophagy in Tumorigenesis and Cancer Treatment pancreatic cancers (Khan et al., 2013). For example, Chen et al. (2014) found that hypoxia-induced MIR155 could target multiple players in mTOR signaling, and enforced expression of MIR155 could increase autophagic activity in human nasopharyngeal cancer and cervical cancer cells. Furthermore, by downregulating mTOR signaling, MIR155 also attenuates cell proliferation and induces G(1)/S cell cycle arrest. The PI3K-AKT-mTOR pathway is a well established driver of cancer in humans, and therefore blocking different nodes of the pathway is a relevant treatment strategy for human malignancies.

Cass III PI3K(Vps34 in yeast) complex signaling pathway

The Vps34 complex is essential for autophagosome formation and maturation. The Vps34 complex generates PtdIns3P, which may allow recruitment of certain ATG components to the phagophore. There are multiple Vps34 complexes, each of which contains the Vps34 lipid kinase, the Vps15 regulatory kinase (p150 in mammals) and ATG6 (BECLIN 1 in mammals). During phagophore formation, the Vps34 complex contains ATG14 (ATG14L in mammals), whereas during maturation into an autolysosome in mammals the complex includes UVRAG. Additional modulating proteins in mammalian cells include AMBRA1, BIF1, RUBICON and HMGB1 (Jaber et al., 2013) (Figure 3).

Emerging evidence suggests that submembers of Vps34 are involved in tumorigenesis. This is especially true for Beclin-1, a phylogenetically conserved protein that is essential for autophagy. In fact, the first association between autophagy and cancer was the landmark discovery of Beclin-1, which is also a haploinsufficient tumor suppressor (Mizushima et al., 2008). In 1999, Liang et al. (1999) used gene transfer techniques in human MCF7 breast carcinoma cells and found that the autophagy promoting activity of Beclin-1 in these cells was associated with inhibition of cellular proliferation. Furthermore, increasing studies show that the Beclin-1 locus (17q21) is frequently subjected to monoallelic deletions in human breast, ovarian, and prostate cancers as well as in brain tumors (Karantza-Wadsworth et al., 2007; DiPaola et al., 2008; Shen et al., 2008). Beclin-1 could be mediated to influence autophagy, tumor progression and chemoresistance. Wei et al. (2013) showed that active epidermal growth factor receptor (EGFR) bound Beclin-1 and led to multisite tyrosine phosphorylation, enhance binding to inhibitors and decrease Beclin-1 associated VPS34 kinase activity. In non-small-cell lung carcinoma tumor xenografts, the expression of a tyrosine phosphomimetic Beclin-1mutant leads to reduced autophagy, enhanced tumor growth and dedifferentiation.

Further study demonstrated that Beclin-mediated tumor suppress involved a Beclin-1 domain. Through this domain, Beclin-1 directly interact with UVRAG and this interaction is purported to promote binding of $\mathrm{Vps} 34$ to Beclin-1. In addition, Bif-1, also known as SH3GLB1 or endophilin B1, was originally discovered as a Bax-binding protein, interacts with Beclin-1 via UVRAG and promotes Vps34 activation and autophagosome formation. In vivo, UVRAG has been reported to suppress cell proliferation and tumorigenesis, and monoallelic deletions or mutations 
Dong-Wei Xu et al

in UVRAG occur in numerous human malignancies (Sun et al., 2009; Takahashi et al., 2009). Furthermore, reduction of Bif-1 expression was observed in gastric carcinomas, invasive urinary bladder, and gallbladder cancers, and mantle cell lymphomas (Lee et al., 2006; Kim et al., 2008; Eisenberg-Lerner and Kimchi, 2009).

\section{ATG12 and LC3 conjugation system}

Both ATG12 conjugation and LC3 modification (ATG8 lipidation in yeast) have been reported to take part in the dynamic process of autophagosome formation (Figure 4); ATG12 conjugation is essential for the formation of preautophagosomes, and LC3 modification is necessary for the formation of autophagosomes (Sun et al., 2009). Recent studies suggest that several key regulators of autophagosome in these two conjugation systems, ATG12 and LC3 conjugations, especially those also associated with apoptosis, are correlated with tumorigenesis. For example, ATG5, playing a role in ATG12 conjugation in the procedure of autophagosome membrane elongation and completion, is reported to interact with Bcl-xL. Kang et al. (2009) reported that frameshift mutations of ATG5 in gastric and colorectal cancers may contribute to cancer development by dysregulating the autophagy process. Lee et al. (2009) recently demonstrated that cellular and viral FLICE-like inhibitor protein (FLIPs), which protect cells from apoptosis mediated by death receptors, limit the ATG3-mediated step of LC3 modification to regulate autophagosome biogenesis. Nevertheless, the precise mechanism linking autophagy-related conjugations and tumorigenesis is still unknown and requires further investigation.

\section{p53-mediated signaling pathway}

p53, a typical tumor suppressor and its mutation could be observed in over $50 \%$ human tumor cells(Soussi et al., 2007). Mutant p53 gene can be detected in non-small cell lung cancer and cannot be found in normal lung tissues through immunohistochemistry SP method. The mutation of p53 may be an important molecular basis of lung cancer' occurrence and development (Zhang et al., 2014). p53 plays double-acting role in regulation of autophagy. In nucleus, genotoxic stress caused by DNA-damaging agents induces p53-dependent autophagy. Similarly, oncogenic activation, simulated by forced expression of ARF or p53, induces autophagy in human cancer cells. The mechanisms of p53-dependent induction of autophagy are still incompletely understood, but are thought to involve both transcription-independent functions (for example, AMPK activation), as well as transcriptiondependent functions (for example, upregulation of mTOR inhibitors, PTEN and TSC1, or the p53-regulated autophagy and cell death gene, DRAM). In some cases, p53-induced autophagy may lead to cell death and this can be blocked by DRAM siRNA8. However, in cmycdriven lymphomas, p53-mediated autophagy increases cell survival, as blockade of autophagosomal maturation enhances p53-mediated tumour regression and tumour-cell death. These seemingly disparate effects of p53-mediated autophagy on life and death decisions of the cell may be cell-type or stimulus-specific, and/or reflect the activation

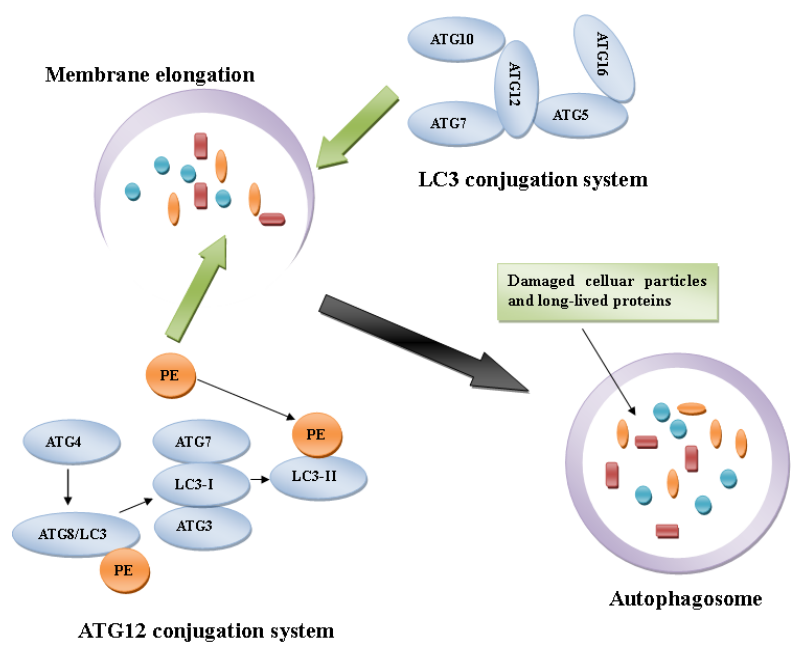

Figure 4. ATG12 and LC3 Conjugation System. During the formation of mammalian autophagosomes, two ubiquitylation-like modifications are required, Atg12conjugation and LC3-modification. LC3 is an autophagosomal ortholog of yeast Atg8. A lipidated form of LC3, LC3-II, has been shown to be an autophagosomal marker in mammals. As the autophagosome matures, the ubiquitin-like protein ATG12 is covalently conjugated to ATG5 through the action of E1- and E2-like proteins ATG7 and ATG10, respectively. ATG12-ATG5ATG16 may act as an E3 ligase for ATG8-PE conjugation, and may dictate the site of conjugation. sequently, the ATG12ATG5 dimer and ATG8-PE assemble and are recruited to the autophagosomal membrane via interaction with ATG16

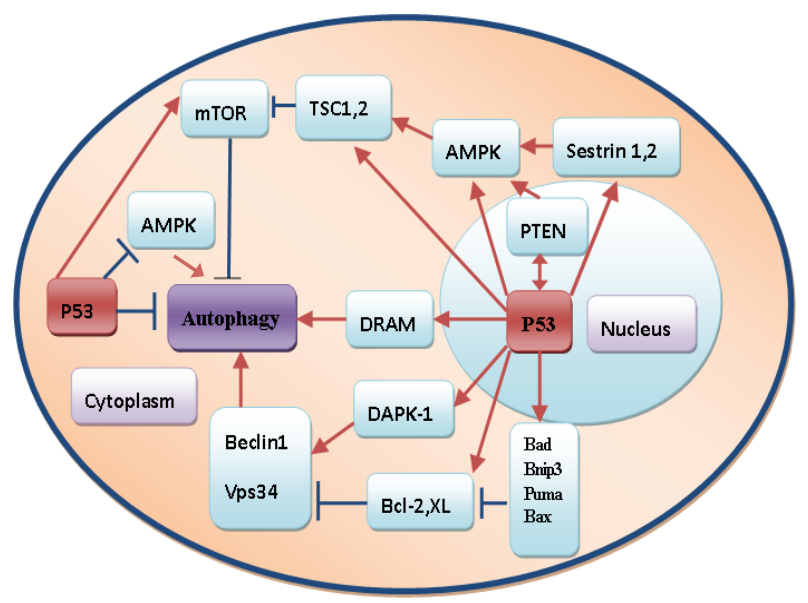

Figure 5. Dual Regulation of p53 in Cell Autophagy

of a different constellation of p53 signals (Levine et al., 2008; O'Prey et al., 2009). However, p53 was able to inhibit autophagy in enucleated cells and cytoplasm, and the inhibition of p53 could induce autophagy. The mechanisms may be the mTOR pathway could not be activated efficiently after $\mathrm{p} 53$ is suppressed (Tasdemir et al., 2008). The Dual regulation of p53 in cell autophagy is shown in Figure 5 (D'Amelio et al., 2009; Maiuri et al., 2009; Sui et al., 2011; White et al., 2011).

\section{Other signaling pathways}

The nuclear fac tor-kappa B (NF- $x \mathrm{~B})$ system is a critical signaling pathway induced to defend cells from cellular damage and environmental danger (Karin, 2006; Van Waes , 2007). NF- $x$ B activation mediates autophagy 
repression through effects on mTOR complex in tumor necrosis factor-alpha (TNF- $\alpha$ )-treated Ewing sarcoma cells (Djavaheri-Mergny et al., 2006). In addition to its role in repressing autophagy, NF- $x \mathrm{~B}$ could activate autophagy that the NF- $\varkappa$ B family member p65/RelA (v-rel reticuloendotheliosis viral oncogene homolog A) upregulated Beclin-1 mRNA and protein levels in different cellular systems (Copetti et al., 2009).

Reactive oxygen species (ROS), as some other signaling molecules, could regulate the activity of ATG4 and induce autophagy in starvation. Starvation stimulates formation of ROS, specifically $\mathrm{H} 2 \mathrm{O} 2$. These oxidative conditions are essential for autophagy, as treatment with antioxidative agents abolished the formation of autophagosomes and the consequent degradation of proteins. The cysteine protease HsATG4 as a direct target for oxidation by $\mathrm{H} 2 \mathrm{O} 2$, and specify a cysteine residue located near the HsATG4 catalytic site as a critical for this regulation. Expression of this regulatory mutant prevented the formation of autophagosomes in cells, thus providing a molecular mechanism for redox regulation of the autophagic process (Scherz-Shouval et al., 2007).

Retinoblastoma protein (RB), a key tumor suppressor and $\mathrm{RB}$ binding to $\mathrm{E} 2$ transcription factor $(\mathrm{E} 2 \mathrm{~F})$ is required for autophagy induction and E2F1 antagonizes RBinduced autophagy, leading to apoptosis. Downregulation of E2F1 in cells results in high levels of autophagy. RB could induce autophagy by repressing E2F1 activity (Jiang et al., 2010).

\section{Roles of Autophagy in Tumorigenesis}

Autophagy is a lysosomal degradation pathway that acts as a dynamic regulator of tumorigenesis. Specifically, autophagy has been shown to impede early cancer development while facilitating advanced tumor progression (Kenific et al., 2014).

The "pro death" function of autophagy could inhibit the formation and development of tumor. In the process of the formation of tumor cells, lack of autophagy may cause energy imbalance and the quality control deviation of proteins and organelles, which can lead to cell damage caused by metabolic stress. Stimulating the DNA damage response and genome unstable expression is main performance, and these could promote tumor occurrence and development. In recent years, a number of specific genes about autophagy were found missing in human malignant tumor (Levine, 2007; Shi et al., 2013). Activated genes in malignant tumor such as PI3K and AKT can prevent autophagy (Ahn et al., 2007; Maiuri et al., 2009). In mouse models, the inactivation of specific genes about autophagy such as Beclin1, ATG5 can lead to an increased incidence of cancer, and up regulate these genes can inhibit tumor formation (Yorimitsu et al., 2005; Rosenfeldt et al., 2011).

The "pro survival" function of autophagy could promote tumor cells survival in stress. Tumor cells induced autophagy and produces response to metabolic stress. Multiple tumor suppressor genes in human malignant tumors such as p53 and PTEN often mutate, which start the occurrence of autophagy. In addition, autophagy is confined to the metabolic stress areas in solid tumors (Degenhardt et al., 2006), and autophagy defects in these areas reduce the survival rate of tumor cells, which is associated with the increase of cell death and inflammation (White et al., 2010). Recent studies have uncovered several tumor-promoting functions for autophagy; these include the maintenance of multiple metabolic pathways critical for aggressive tumor growth and the promotion of tumor cell survival downstream of the unfolded protein response. Furthermore, autophagy supports anoikis resistance and cancer cell invasion. At the same time, because autophagy cargo receptors, which are essential for selective autophagy, lie upstream of diverse cancer-promoting signaling pathways, they may profoundly influence how alterations in autophagy affect tumor development (Kenific et al., 2014).

The dual function of autophagy make it complicated on tumor treatment. The "pro death" function can kill tumor cells spontaneously, or to help radiation and chemotherapy kill tumor cells. The "pro survival" function could help the tumor cell survival in nutrition deficiency environment and resist ionizing radiation and chemotherapy. Therefore, this contradiction may lead to more intense debate about the relationship between autophagy and cancer: whether autophagy is good or bad for tumor.

\section{Autophagy as a Tumor-suppressing Mechanism}

Deficiencies in autophagy lead to the accumulation of damaged macromolecules and organelles (particularly mitochondria), subsequently inducing oxidative stress, DNA damage and chromatin instability. Thus, autophagic defects are ultimately associated with the accumulation of oncogenic mutations and increased tumor susceptibility. Some of the most important evidence for the role of autophagy in tumor suppression comes from studies on the Bcl-2-interacting protein, Beclin1 (BECN1, also called ATG6), and much study showed that allelic loss of beclin1 promotes mammary tumorigenesis and activation of the DNA damage response in tumors in vivo (KarantzaWadsworth et al., 2007). Furthermore, autophagy has been shown to reduce intratumoral necrosis and local inflammation (Degenhardt et al., 2006). Collectively, these results support the contention that autophagy plays a tumor-suppressing role in cancer, and suggest that reduced autophagic activity may constitute a hallmark of cancer (Hanahan et al., 2011).

At present, autophagy molecular mechanism of inhibiting tumor is not completely understood. More and more evidences suggest that autophagy antitumor effect may be associated with its potential "pro death" and "pro survival" function. The lack of monoallelic Beclin1 or biallelic ATG5 may lead to epithelial tumors, but it will not reduce cell death (Yorimitsu et al., 2005; Rosenfeldt et al., 2009).

Research suggests (Du et al., 2010) that tumor cells cannot die through apoptosis in the condition of metabolic stress; however, autophagy can prevent cell death at this time. Local inflammation may increase once cell necrosis happen, which will increase tumor growth. ATG genes' 
missing may increase genomic instability in metabolic stress condition, which leads to proto-oncogene activation and tumor process promotion (Rufini et al., 2011).

In normal cells, it is not clear whether autophagy can make gene expression stable and plays an important role in inhibiting tumor. Another possibility is that autophagy plays a direct role in negative growth. Research shows that enhancing Beclinl expression does not lead to increased cell death, and can also slow tumor cell line proliferation, reduce the cell cycle regulatory proteins $\mathrm{E}$ and phosphorylation of Rb (Koneri et al., 2007). Beelinl, on the other hand, mammary gland epithelial cells and spleen lymphocytes in mice were greatly expanded when monoallelic Beclin1 missing (Qu et al., 2003).

Also studies have shown that although autophagy has "pro survival" effect, autophagy can avoid inappropriate cell differentiation when tumor cells in metabolic stress. In Beclinl +/- mice, mammary epithelial cells which are in dormant state at usual times can proliferate spontaneously. This suggests that autophagy can coordinate the relationship between environmental signal and the cells enter or maintain in G0. Although it is not clear whether cell survival/death is associated with the tumor inhibition effect of autophagy, but this phenomenon is a very important entry point to tumor treatment in the future. Recently, some scholars questioned the effect of sensitizing tumor cells to cytotoxic therapy by blocking autophagy. One study attributes that the effect of regulating the autophagy depends on the tumor intrinsic properties and the nature of the combination cytotoxic drugs. Thus, although regulating autophagy may affect tumor growth, metastasis and treatment resistance, the unique attributes of tumor and the cytotoxic drugs we choose may decide the result of treatment (Yang et al., 2011).

In fact, in mice lymphoma induced by c-Myc, chloroquine can reduce autophagosomes degradation and enhance p53 or DNA alkylating agent' function of inducing tumor cell death and regression. That means autophagy serves as a potential "pro survival" and antitumor effect in cancer chemotherapy. Chloroquine is effective to both tumor cells and host immune system (Apetoh et al., 2007), so we cannot deduce that its anti-tumor effect is only mediated by inhibiting autophagy. We need more invivo test to compare the advantages of blocking tumor cell survival pathway and the disadvantages of blocking tumor suppressor pathway.

\section{The Role of Autophagy in Cancer Treatment}

For all eukaryotes, autophagy specific gene can promote the survival of normal cells in times of nutrient scarcity. Autophagy also could improve the survival ability of tumor cells which is facing lack of oxygen or metabolic stress because of poor blood supply. In some huge tumor, abnormal vascular system will eventually lead to low oxygen, low $\mathrm{pH}$ and nutritional deficiency in the center of the tumor cells, and these metabolic stress area is where autophagy occurs (Karantza-Wadsworth et al., 2007). Therefore, tumor cells could rely on autophagy to suvive under the condition of lack of energy and nutrition, and is likely to degrade damaged mitochondria and other organelles through lysosome. Thus, it can inhibit the activation of tumor suppressor gene or buffer treatment stress to promote the survival of tumor cells. This means we can block autophagy in tumor treatment; on the contrary, the lack of autophagy will lead to cell death, chronic inflammation and genomic instability, which makes tumor easy to happen, so reducing cell damage by autophagy is a new tumor suppressor mechanism (Maiuri et al., 2009). Recent years studies showed that chloroquine as an autophagy inhibitor did not only inhibit autophagy but also inhibit the function of lysosome, so it may be beneficial for tumor therapy (Rosenfeldt et al., 2009). However, excessive autophagy or autophagy happens in cells lack of apoptosis mechanism, could also cause cell death (Ahn et al., 2007). We cannot easily get an conclusion that tumor cells can be killed through turning on or off the autophagy pathway.

In addition, the residual or metastatic tumor cell could survive against metabolic stress through autophagy, and it may be a key mechanism of tumor recurrence (AguirreGhiso, 2006). Thus, inhibition of autophagy has great significance in tumor cells intolerance low metabolic status and improving prognosis.

Now autophagy has been recognized as an important regulator of cancer development and progression and a key factor in determining tumor cell sensitivity to anticancer therapy. Numerous conventional and experimental anti-tumor strategies, including combination therapies with autophagy regulators, have shown that targeting autophagy is a beneficial approach to cancer therapy ( $\mathrm{Li}$ et al., 2011).

Autophagy plays a complicated role in tumorigenesis and treatment responsiveness. It can be tumor-suppressing during the early stages of tumorigenesis, as reduced autophagy is found in tumor cells and may be associated with malignant transformation. In this case, induction of autophagy would seem to be beneficial for cancer prevention. In established tumors, however, autophagy can be tumor-promoting, and cancer cells can use enhanced autophagy to survive under metabolic and therapeutic stress. The pharmacological and/or genetic inhibition of autophagy was recently shown to sensitize cancer cells to the lethal effects of various cancer therapies, including chemotherapy, radiotherapy and targeted therapies, suggesting that suppression of the autophagic pathway may represent a valuable sensitizing strategy for cancer treatments. In contrast, excessive stimulation of autophagy may also provide a therapeutic strategy for treating resistant cancer cells having high apoptotic thresholds. In order for us to develop successful autophagy-modulating strategies against cancer, we need to better understand how the roles of autophagy differ depending on the tumor stage, cell type and/or genetic factors, and we need to determine how specific pathways of autophagy are activated or inhibited by the various anti-cancer therapies.

In short, autophagy play an complex role in tumor biology for its two sides of effects on tumor cells. As a new theory in tumor treatment field, with the further research of the mechanism and function of autophagy, exploring tumor treatment strategy on the level of autophagy has broad prospects. 


\section{Plant Natural Drugs Play Anti-cancer Effects Through Autophagy}

Natural compounds derived from plant sources are well characterized as possessing a wide variety of remarkable anti-tumour properties, such as modulating apoptosis and autophagy. Here are some anti-tumor natural compounds, and recent studies showed that their anti-tumor effects may be associated with autophagy.

Resveratrol, a natural phytoalexin present in grapes, nuts, and red wine, has antineoplastic activities (Opipari et al., 2004), and it is notable that Resveratrol can inhibit initiation and growth of tumours in a wide range of cancer models. Research proves that resveratrol could induce autophagy through death-associated protein kinase 1 (DAPK1) in human dermal fibroblasts under normal culture conditions (Choi et al., 2013); also, resveratrol could protect $\mathrm{H} 2 \mathrm{O} 2$-treated $\mathrm{H} 9 \mathrm{c} 2$ cells by upregulating autophagy via the p38 MAPK pathway (Lv et al., 2012). Curcumin, derived from turmeric, has been shown to demonstrate activity in a wide range of biological functions, particularly against cancer (Pal et al., 2005). Moreover, curcumin has been reported to regulate autophagy, resulting in inhibition of several types of cancer cell proliferation. Curcumin can significantly decrease the expression of PI3K, phosphorylated Akt and rapamycin (mTOR) at protein levels, respectively. It suggests that curcumin induces autophagy by downregulating PI3K/ Akt/mTOR signaling pathway (Wang et al., 2014). Cucurbitacin, iisolated from the root of Hemsleya amabilis, is another anti-tumor natural compound. Cells treatment with cucurbitacin I up-regulated Beclin 1 and triggered autophagosome formation and accumulation as well as conversion of LC3-I to LC3-II. Activation of the AMP-activated protein kinase/mammalian target of rapamycin/p70S6K pathway, but not the PI3K/AKT pathway, occurred in autophagy induced by cucurbitacin I, which was accompanied by decreased hypoxia-inducible factor $1 \alpha$ (Yuan et al., 2014). Cucurbitacin family, such as cucurbitacin IIa, cucurbitacin B, cucurbitacin E have some analogous effects (Zhu et al., 2012; He et al., 2013).

There are many other natural compounds from plants, like Matrine, palmitic acid, lycopene, capsaicin, deguelin and quercetin have been found to induce cancer cell death by regulation of autophagy (Choi et al., 2010; Wang et al., 2013; Yang et al., 2013; Chen et al., 2014; Chen et al., 2014; Tu et al., 2014). With the accumulating exploration of molecular mechanisms of plant natural compound activity, they have been widely used as candidate antitumour agents; more importantly, some have been further applied to preclinical and clinical cancer therapies.

\section{Conclusions}

In summary, autophagy is a ubiquitous process in eukaryotic cells that results in the breakdown of cytoplasm within the lysosome in response to stress conditions, which allows the cell to adapt to environmental and/or developmental changes. Increasing evidence lndicates that autophagy is associated with a number of pathological process, including cancer. We have already found severaI
Importance of Autophagy in Tumorigenesis and Cancer Treatment key autophagic mediators (eg, Beclin1, UVRAG, Class III and I PI3K, mTOR, and p53) that play important roles in autophagic signaling networks in cancer. However, Cancer is a complex, multi-step human disease that is closely related to the Janus of autophagy. Currently, much work should be done to determine the molecular mechanisms of autophay in cancer, to define how the crucial modulators of autophagy in cancer impacts cancer initiation and progression, and to elucidate why targeting autophagic signaling pathways is promising for cancer therapeutics. The present research shows that appropriate modification of autophagy, that is, suppression of cell-protective autophagy or enhancement of cell-killing autophagy, can augment cytotoxicity caused by anti-cancer therapy. Hence, modulating autophagy will open new areas for cancer therapy.

\section{References}

Aguirre-Ghiso JA (2006). The problem of cancer dormancy: understanding the basic mechanisms and identifying therapeutic opportunities. Cell Cycle, 5, 1740-3.

Ahn CH, Jeong EG, Lee JW, et al (2007). Expression of beclin-1, an autophagy-related protein, in gastric and colorectal cancers. APMIS, 115, 1344-9.

Apetoh L, Ghiringhelli F, Tesniere A, et al (2007). Toll-like receptor 4-dependent contribution of the immune system to anticancer chemotherapy and radiotherapy. Nat Med, 13, 1050-9.

Chen BL, Wang LT, Huang KH, et al (2014). Quercetin attenuates renal ischemia/reperfusion injury via an activation of AMPactivated protein kinase-regulated autophagy pathway.J Nutr Biochem, 25, 1226-34

Chen F, Sun ZW, Ye LF, et al (2014). Lycopene protects against apoptosis in hypoxia/reoxygenationinduced $\mathrm{H} 9 \mathrm{C} 2$ myocardioblast cells through increased autophagy. Mol Med Rep, 11, 1358-65.

Choi CH, Jung YK, Oh SH (2010). Autophagy induction by capsaicin in malignant human breast cells is modulated by p38 and extracellular signal-regulated mitogen-activated protein kinases and retards cell death by suppressing endoplasmic reticulum stress-mediated apoptosis. Mol Pharmacol, 78, 114-25.

Choi MS, Kim Y, Jung JY, et al (2013). Resveratrol induces autophagy through death-associated protein kinase 1 (DAPK1) in human dermal fibroblasts under normal culture conditions. Exp Dermatol, 22, 491-4.

Copetti T, Bertoli C, Dalla E, Demarchi F, Schneider C(2009). p65/RelA modulates BECN1 transcription and autophagy. Mol Cell Biol, 29, 2594-608.

D'Amelio M, Cecconi F (2009). A novel player in the p53mediated autophagy: Sestrin2. Cell Cycle, 8, 1467.

De Duve C, Pressman BC, Gianetto R, Wattiaux R, Appelmans F (1955). Tissue fractionation studies. 6. Intracellular distribution patterns of enzymes in rat-liver tissue. Biochem $J$, 60, 604-17.

Degenhardt K, Mathew R, Beaudoin B, et al (2006). Autophagy promotes tumor cell survival and restricts necrosis, inflammation, and tumorigenesis. Cancer Cell, 10, 51-64.

DiPaola RS, Dvorzhinski D, Thalasila A, et al (2008). Therapeutic starvation and autophagy in prostate cancer: a new paradigm for targeting metabolism in cancer therapy. Prostate, 68, 1743-52.

Djavaheri-Mergny M, Amelotti M, Mathieu J, et al (2006). NF-kappaB activation represses tumor necrosis factor-alpha- 
induced autophagy. J Biol Chem, 281, 30373-82.

Du J, An J, Wei N, et al (2010). Increased resistance to LPSinduced myocardial dysfunction in the Brown Norway rats versus Dahl S rats: roles of inflammatory cytokines and nuclear factor kappaB pathway. Shock, 33, 332-6.

Eisenberg-Lerner A, Kimchi A (2009). The paradox of autophagy and its implication in cancer etiology and therapy. Apoptosis, 14,376-91.

Galluzzi L, Vitale I, Abrams JM, et al (2012). Molecular definitions of cell death subroutines: recommendations of the nomenclature committee on cell death 2012. Cell Death Differ, 19, 107-20.

Geng J, Baba M, Nair U, Klionsky DJ (2008). Quantitative analysis of autophagy-related protein stoichiometry by fluorescence microscopy. J Cell Biol, 182, 129-40.

Ghayad SE, Cohen PA(2010). Inhibitors of the PI3K/Akt $/ \mathrm{mTOR}$ pathway: new hope for breast cancer patients. Recent Pat Anticancer Drug Discov, 5, 29-57.

Gozuacik D, Kimchi A (2004). Autophagy as a cell death and tumor suppressor mechanism. Oncogene, 23, 2891-906.

Hanahan D, Weinberg RA (2011). Hallmarks of cancer: the next generation. Cell, 144, 646-74.

He J, Wang Y, Xu LH et al (2013). Cucurbitacin IIa induces caspase-3-dependent apoptosis and enhances autophagy in lipopolysaccharide-stimulated RAW 264.7 macrophages. Int Immunopharmacol, 16, 27-34.

Hotchkiss RS, Strasser A, McDunn JE, Swanson PE (2009). Cell death. $N$ Engl J Med, 361, 1570-83.

Huang J, Klionsky DJ (2007). Autophagy and human disease. Cell Cycle, 6, 1837-49.

Inoki K, Guan KL (2009). Tuberous sclerosis complex, implication from a rare genetic disease to common cancer treatment. Hum Mol Genet, 18, 94-100.

Jaber N, Zong WX (2013). Class III PI3K Vps34: essential roles in autophagy, endocytosis, and heart and liver function. Ann N Y Acad Sci, 1280, 48-51.

Jiang H, Martin V, Gomez-Manzano C, et al (2010). The RB-E2F1 pathway regulates autophagy. Cancer Res, 70, 7882-93.

Kang MR, Kim MS, Oh JE, et al (2009). Frameshift mutations of autophagy-related genes ATG2B, ATG5, ATG9B and ATG12 in gastric and colorectal cancers with microsatellite instability. J Pathol, 217, 702-6.

Karantza-Wadsworth V, Patel S, Kravchuk O, et al (2007). Autophagy mitigates metabolic stress and genome damage in mammary tumorigenesis. Genes Dev, 21, 1621-35.

Karantza-Wadsworth V, White E (2007). Role of autophagy in breast cancer. Autophagy, 3, 610-3.

Karin M (2006). Nuclear factor-kappaB in cancer development and progression. Nature, 441, 431-6.

Kenific CM, Debnath J (2014). Cellular and metabolic functions for autophagy in cancer cells. Trends Cell Biol, 25, 37-45.

Khan KH, Yap TA, Yan L, Cunningham D (2013). Targeting the PI3K-AKT-mTOR signaling network in cancer. Chin J Cancer, 32, 253-65.

Kim SY, Oh YL, Kim KM, et al (2008). Decreased expression of Bax-interacting factor-1 (Bif-1) in invasive urinary bladder and gallbladder cancers. Pathology, 40, 553-7.

Klionsky DJ(2005). The molecular machinery of autophagy: unanswered questions. J Cell Sci, 118, 7-18.

Klionsky DJ (2007). Autophagy: from phenomenology to molecular understanding in less than a decade. Nat Rev Mol Cell Biol, 8, 931-7.

Klionsky DJ (2014). Citing recent declines in the discovery of new ATG genes, some scientists now suggest that the end of autophagy research may be within sight. Autophagy, 10, 715-6.
Klionsky DJ, Cregg JM, Dunn WA, et al (2003). A unified nomenclature for yeast autophagy-related genes. Dev Cell, 5, 539-45.

Koneri K, Goi T, Hirono Y, Katayama K, Yamaguchi A (2007). Beclin 1 gene inhibits tumor growth in colon cancer cell lines. Anticancer Res, 27, 1453-7.

Lee JS, Li Q, Lee JY, et al (2009). FLIP-mediated autophagy regulation in cell death control. Nat Cell Biol, 11, 1355-62.

Lee JW, Jeong EG, Soung YH, et al (2006). Decreased expression of tumour suppressor Bax-interacting factor-1 (Bif-1), a Bax activator, in gastric carcinomas. Pathology, 38, 312-5.

Levine B (2007). Cell biology: autophagy and cancer. Nature, 446, 745-7.

Levine B, Abrams J (2008). p53: The janus of autophagy? Nat Cell Biol, 10, 637-9.

Li J L, Han SL, Fan X (2011). Modulating autophagy: a strategy for cancer therapy. Chin J Cancer, 30, 655-68.

Liang XH, Jackson S, Seaman M, et al (1999). Induction of autophagy and inhibition of tumorigenesis by beclin 1 . Nature, 402, 672-6.

Lv XC, Zhou HY (2012). Resveratrol protects H9c2 embryonic rat heart derived cells from oxidative stress by inducing autophagy: role of p38 mitogen-activated protein kinase. Can J Physiol Pharmacol, 90, 655-62.

Maiuri MC, Malik SA, Morselli E, et al (2009). Stimulation of autophagy by the p53 target gene Sestrin2. Cell Cycle, 8, 1571-6.

Maiuri MC, Tasdemir E, Criollo A, et al (2009). Control of autophagy by oncogenes and tumor suppressor genes. Cell Death Differ, 16, 87-93.

Massey A, Kiffin R, Cuervo AM (2004). Pathophysiology of chaperone-mediated autophagy. Int J Biochem Cell Biol, 36, 2420-34.

Mizushima N, Levine B, Cuervo AM, Klionsky DJ (2008). Autophagy fights disease through cellular self-digestion. Nature, 451, 1069-75.

Morselli E, Galluzzi L, Kepp O, et al (2009). Anti- and protumor functions of autophagy. Biochim Biophys Acta, 1793, 1524-32.

O'Prey J, Skommer J, Wilkinson S, Ryan KM (2009). Analysis of DRAM-related proteins reveals evolutionarily conserved and divergent roles in the control of autophagy. Cell Cycle, 8, 2260-5.

Ohsumi Y (2006). Autophagy related genes in yeast, S. cerevisiae. Tanpakushitsu Kakusan Koso, 51, 1453-6.

Opipari AW, Jr, Tan L, et al (2004). Resveratrol-induced autophagocytosis in ovarian cancer cells. Cancer Res, 64, 696-703.

Pal S, Bhattacharyya S, Choudhuri T, et al (2005). Amelioration of immune cell number depletion and potentiation of depressed detoxification system of tumor-bearing mice by curcumin. Cancer Detect Prev, 29, 470-8.

Qu X, Yu J, Bhagat G, et al (2003). Promotion of tumorigenesis by heterozygous disruption of the beclin 1 autophagy gene. J Clin Invest, 112, 1809-20.

Rosenfeldt MT, Ryan KM (2009). The role of autophagy in tumour development and cancer therapy. Expert Rev Mol Med, 11, 36 .

Rosenfeldt MT, Ryan KM (2011). The multiple roles of autophagy in cancer. Carcinogenesis, 32, 955-63.

Rufini A, Melino G (2011). Cell death pathology: the war against cancer. Biochem Biophys Res Commun, 414, 445-50.

Scherz-Shouval R, Shvets E, Fass E, et al (2007). Reactive oxygen species are essential for autophagy and specifically regulate the activity of Atg4.EMBO J, 26, 1749-60.

Shen Y, Li DD, Wang LL, Deng R, Zhu XF (2008). Decreased expression of autophagy-related proteins in malignant 
epithelial ovarian cancer. Autophagy, 4, 1067-8.

Shi Z, Li CY, Zhao S, et al (2013). A systems biology analysis of autophagy in cancer therapy. Cancer Lett, 337, 149-60.

Soussi T, Wiman KG (2007). Shaping genetic alterations in human cancer: the p53 mutation paradigm. Cancer Cell, 12, 303-12.

Sui X, Jin L, Huang X, et al (2011).p53 signaling and autophagy in cancer: a revolutionary strategy could be developed for cancer treatment. Autophagy, 7, 565-71.

Sun Q, Fan W, Zhong Q (2009). Regulation of Beclin 1 in autophagy. Autophagy, 5, 713-6.

Takahashi Y, Meyerkord CL, Wang HG (2009). Bif-1/endophilin B1: a candidate for crescent driving force in autophagy. Cell Death Differ, 16, 947-55.

Tanida I, Ueno T, Kominami E (2004). LC3 conjugation system in mammalian autophagy. Int $J$ Biochem Cell Biol, 36, 2503-18.

Tasdemir E, Maiuri MC, Galluzzi L, et al (2008). Regulation of autophagy by cytoplasmic p53. Nat Cell Biol, 10, 676-87.

Tu QQ, Zheng RY, Li J, et al (2014). Palmitic acid induces autophagy in hepatocytes via JNK2 activation. Acta Pharmacol Sin, 35, 504-12.

Van Waes C (2007). Nuclear factor-kappaB in development, prevention, and therapy of cancer. Clin Cancer Res, 13, 1076-82.

Wang C,Zhang X, Teng Z,Zhang T,Li Y (2014). Downregulation of PI3K/Akt/mTOR signaling pathway in curcumin-induced autophagy in APP/PS1 double transgenic mice. Eur $J$ Pharmacol, 740, 312-20.

Wang Z, Zhang J, Wang Y, et al (2013). Matrine, a novel autophagy inhibitor, blocks trafficking and the proteolytic activation of lysosomal proteases. Carcinogenesis, $\mathbf{3 4}$, 128-38.

Wei Y, Zou Z, Becker N, et al (2013). EGFR-mediated Beclin 1 phosphorylation in autophagy suppression, tumor progression, and tumor chemoresistance. Cell, 154, 1269-84.

White E, Karp C, Strohecker AM, Guo Y, Mathew R (2010). Role of autophagy in suppression of inflammation and cancer. Curr Opin Cell Biol, 22, 212-7.

White EJ, Martin V, Liu JL, et al (2011). Autophagy regulation in cancer development and therapy. Am J Cancer Res, $\mathbf{1}$, 362-72.

Yang YL, Ji C, Bi ZG, et al (2013). Deguelin induces both apoptosis and autophagy in cultured head and neck squamous cell carcinoma cells. PLoS One, $\mathbf{8}, 54736$.

Yang Z, Klionsky DJ (2009). An overview of the molecular mechanism of autophagy. Curr Top Microbiol Immunol, 335, 1-32.

Yang ZJ, Chee CE, Huang S, Sinicrope F (2011). Autophagy modulation for cancer therapy. Cancer Biol Ther, 11, 169-76.

Yorimitsu T, Klionsky DJ (2005). Autophagy: molecular machinery for self-eating. Cell Death Differ, 12, 1542-52.

Yuan G, Yan SF, Xue H, et al (2014). Cucurbitacin I induces protective autophagy in glioblastoma in vitro and in vivo.J Biol Chem, 289, 10607-19.

Zhang EB, Yin DD, Sun M, et al (2014). P53-regulated long non-coding RNA TUG1 affects cell proliferation in human non-small cell lung cancer, partly through epigenetically regulating HOXB7 expression. Cell Death Dis, 5, 1243.

Zhu JS, Ouyang DY, Shi ZJ (2012). Cucurbitacin B induces cell cycle arrest, apoptosis and autophagy associated with $\mathrm{G}$ actin reduction and persistent activation of cofilin in Jurkat cells. Pharmacology, 89, 348-56. 